\title{
Two Positive Solutions of a Quasilinear Elliptic Dirichlet Problem
}

\author{
C.A. Stuart
}

\begin{abstract}
For a class of second order quasilinear elliptic equations we establish the existence of two non-negative weak solutions of the Dirichlet problem on a bounded domain, $\Omega$. Solutions of the boundary value problem are critical points of $C^{1}$-functional on $H_{0}^{1}(\Omega)$. One solution is a local minimum and the other is of mountain pass type.
\end{abstract}

Mathematics Subject Classification (2010). Primary 35J62; Secondary 35J60.

Keywords. Mountain pass solution, Cerami sequence.

\section{Introduction}

Let $\Omega$ be a bounded open subset of $\mathbb{R}^{N}$ and let $X$ denote the Sobolev space $H_{0}^{1}(\Omega)$ with the norm $\|u\|=\left\{\int_{\Omega}|\nabla u|^{2} d x\right\}^{1 / 2}$. The usual norm on $L^{p}(\Omega)$ is denoted by $|\cdot|_{p}$.

In this paper we consider critical points of the functional $\Phi_{\lambda, h}: X \rightarrow \mathbb{R}$ defined by

$$
\Phi_{\lambda, h}(u)=\int_{\Omega} \Gamma\left(\frac{1}{2}\left[u^{2}+|\nabla u|^{2}\right]\right) d x-\frac{\lambda}{2} \int_{\Omega} u^{2} d x-\int_{\Omega} u h d x
$$

where $\lambda \in \mathbb{R}$ and the functions $\Gamma$ and $h$ satisfy the following conditions.

(g1) (a) $\Gamma \in C^{1}([0, \infty), \mathbb{R})$ with $\Gamma(0)=0, \gamma=\Gamma^{\prime}$ non-increasing on $[0, \infty)$ and $\gamma(\infty)=\lim _{t \rightarrow \infty} \gamma(t)>0$.

(b) $h \in L^{2}(\Omega)$ and $h \geq 0$ a.e. on $\Omega$.

The idea of studying the critical points of (1.1) under the hypotheses (g1) came from earlier work with H.-S. Zhou $[11,12]$ where a more complicated functional with some similar features was treated on $(0, \infty)$ instead of on a bounded subset of $\mathbb{R}^{N}$. Other types of quasilinear equations have been discussed using min-max methods in $[2,13]$.

Proposition 3.1 shows hypothesis (g1) ensures that $\Phi_{\lambda, h} \in C^{1}(X, \mathbb{R})$ for all $\lambda \in \mathbb{R}$ and critical points of $\Phi_{\lambda, h}$ correspond to weak solutions of the Dirichlet 
problem

$$
\begin{array}{cc}
-\nabla \cdot\left[\gamma\left(\frac{1}{2}\left[u^{2}+|\nabla u|^{2}\right]\right) \nabla u\right]+\gamma\left(\frac{1}{2}\left[u^{2}+|\nabla u|^{2}\right]\right) u=\lambda u+h & \text { in } \Omega \\
u=0 & \text { on } \partial \Omega .
\end{array}
$$

Clearly $u \equiv 0$ is a solution of this problem for all $\lambda$ when $h \equiv 0$.

If $\gamma(0)=\gamma(\infty)$ and $h \equiv 0, \gamma$ is constant and (1.2) becomes the linear eigenvalue problem

$$
-\Delta u+u=\frac{\lambda}{\gamma(\infty)} u \text { in } \Omega, \quad u=0 \text { on } \partial \Omega .
$$

The non-zero critical points of (1.1) are precisely the eigenfunctions of the Laplacian on $\Omega$ with the Dirichlet boundary condition. In particular, setting

$$
\lambda_{1}=\inf \left\{\int_{\Omega}|\nabla u|^{2}: u \in X=H_{0}^{1}(\Omega) \text { with } \int_{\Omega} u^{2} d x=1\right\},
$$

it is well-known that $\lambda_{1}>0$ is the lowest eigenvalue and there exists an eigenfunction $\phi \in H_{0}^{1}(\Omega) \cap C^{2}(\Omega)$ with $\phi>0$ and $-\Delta \phi=\lambda_{1} \phi$ in $\Omega$. Furthermore, $\lambda_{1}$ is a simple eigenvalue and all eigenfunctions corresponding to higher eigenvalues change sign on $\Omega$. Hence, when $\gamma(\infty)=\gamma(0)$ and $h \equiv 0,(1.2)$ has a positive solution if and only if $\lambda=\gamma(\infty)\left[1+\lambda_{1}\right]$. In fact, the set of all positive solutions is $\{t \phi: t>0\}$. When $\gamma(\infty)=\gamma(0)$ and $h \not \equiv 0,(1.2)$ has a positive solution if and only if $\lambda<\gamma(\infty)\left[1+\lambda_{1}\right]$.

From now on we deal with the case $\gamma(\infty)<\gamma(0)$ where (1.2) has a quasilinear structure and (g1) is no longer sufficient to ensure its ellipticity. Indeed, setting $F(z, p)=\Gamma\left(\frac{1}{2}\left[z^{2}+|p|^{2}\right]\right)$ for $z \in \mathbb{R}$ and $p \in \mathbb{R}^{N}$, it is well-known, see Chapter 10 of [7], that the ellipticity of (1.2) is equivalent to the convexity of $F(z, p)$ with respect to $p$ for all $(z, p)$. It is easily seen that this corresponds to the convexity of $g(t)=\Gamma\left(t^{2}\right)$ on $[0, \infty)$. As is shown in an appendix, the following hypothesis ensures the uniform ellipticity of (1.2).

(g2) For $\Gamma$ satisfying (g1) and with $g(t)=\Gamma\left(t^{2}\right)$, there exists $\rho>0$ such that $g(t) \geq g(s)+g^{\prime}(s)(t-s)+\rho(t-s)^{2}$ for all $t, s \geq 0$.

It is easily checked that (g2) is equivalent to the property $\frac{g^{\prime}(t)-g^{\prime}(s)}{t-s} \geq 2 \rho$ for $t>s \geq$ 0 . Thus we see that (g1) requires $\Gamma(t)$ to be a concave function of $t$, whereas (g2) imposes a kind of uniform, strict convexity in $t$ on $\Gamma\left(t^{2}\right)$. As is shown in Section 3, (g2) ensures that $\Phi_{\lambda, h}$ is weakly sequentially lower semi-continuous on $X$, although a weaker form of convexity would suffice for this purpose. The full strength of (g2) is invoked in Section 8.

For our treatment of the homogenous case $h \equiv 0$ the hypotheses (g1) and (g2) are sufficient. However for the inhomogeneous problem, we require one further condition on the function $\Gamma$. Setting $K(t)=\Gamma(t)-\Gamma^{\prime}(t) t=\int_{0}^{t}[\gamma(s)-\gamma(t)] d s$ for $t \geq 0$, it follows from (g1) that

$$
K \in C([0, \infty)), K(0)=0 \text { and } K \text { non-decreasing on }[0, \infty) .
$$

(g3) For $\Gamma$ satisfying (g1), $\lim _{t \rightarrow \infty}\left\{\Gamma(t)-\Gamma^{\prime}(t) t\right\}<\infty$. 
If (g1) holds and $\Gamma \in C^{2}([0, \infty))$, then (g2) is satisfied provided that inf $t \geq 0\{\gamma(t)+$ $\left.2 t \gamma^{\prime}(t)\right\}>0$ and (g3) is satisfied provided that $\int_{0}^{\infty}\left|\gamma^{\prime}(t)\right| t d t<\infty$.

Example Consider $\gamma(t)=A+(1+t)^{-\alpha}$ for $t \geq 0$ where $A>0$ and $\alpha>0$. Then (g1) is satisfied by $\Gamma(t)=\int_{0}^{t} \gamma(s) d s$. For fixed $\alpha$, (g2) holds for large enough values of $A$ and (g3) holds if $\alpha>1$. Here are some typical cases.

For $\alpha=1 / 2, \Gamma(t)=A t+2[\sqrt{1+t}-1]$ and (g2) holds for all $A>0$ whereas (g3) always fails.

For $\alpha=1, \Gamma(t)=A t+\ln (1+t)$ and (g2) holds if and only if $A>1 / 8$ but (g3) is never satisfied.

For $\alpha=3, \Gamma(t)=A t-\frac{1}{2}\left[(1+t)^{-2}-1\right]$ and $(\mathrm{g} 2)$ is satisfied for $A>1 / 3$ whereas (g3) is satisfied for all $A>0$. The value $1 / 3$ is not optimal for obtaining (g2), the sharp condition being $A>5^{4} / 2^{11} \sim 0.3052$.

We can now state the main result of this paper.

Theorem 1.1. Let (g1) and (g2) be satisfied with $\gamma(\infty)<\gamma(0)$ and consider $\lambda$ such that $\gamma(\infty)+\gamma(\infty) \lambda_{1}<\lambda<\gamma(0)+\gamma(\infty) \lambda_{1}$.

(I) For $h \equiv 0, u_{1} \equiv 0$ is a solution of (1.2) with $\Phi_{\lambda, 0}\left(u_{1}\right)=0$ and there is another non-negative weak solution $u_{2} \in H_{0}^{1}(\Omega)$ with $\Phi_{\lambda, 0}\left(u_{2}\right)>0$.

(II) If in addition (g3) is satisfied, there exists $H_{\lambda}>0$ such that $0<|h|_{2}<H_{\lambda}$ ensures that (1.2) has at least two distinct non-negative weak solutions $u_{1}, u_{2} \in$ $H_{0}^{1}(\Omega)$ with $\Phi_{\lambda, h}\left(u_{2}\right)>0>\Phi_{\lambda, h}\left(u_{1}\right)$.

In both parts, $u_{1}$ is a strict local minimum of $\Phi_{\lambda, h}$ whereas $u_{2}$ is characterized by a min-max principle of mountain pass type. Furthermore, $u_{1}$ is not a global minimum since $\inf _{u \in X} \Phi_{\lambda, h}(u)=-\infty$ under our hypotheses.

Proof. This follows from Theorem 4.2 and Corollary 8.2. In Theorem 4.2 the solution $u_{1}$ is obtained as a local minimum of $\Phi_{\lambda, h}$ in a neighbourhood of the origin. For this we assume that $\Gamma$ satisfies (g1) and $(\mathrm{g} 2)$ and that $\lambda<\gamma(0)+\gamma(\infty) \lambda_{1}$. The positivity of $u_{1}$ is deduced from its characterization as a local minimum. Then, with the additional assumption that $\lambda>\gamma(\infty)\left[1+\lambda_{1}\right]$, we show that $\Phi_{\lambda, h}$ has a mountain pass geometry. Referring to a result presented in Section 2, this implies the existence of a sequence of approximate critical points of $\Phi_{\lambda, h}$ having some additional properties. We use these properties to show that the sequence is bounded in $X$, provided that (g3) is satisfied in the case $h \not \equiv 0$. Using the hypothesis (g2), we establish in Corollary 8.2 the existence of a second solution $u_{2}$ as the limit of a subsequence of these approximate critical points. The positivity of $u_{2}$ is a consequence of the localization of the approximating sequence derived from the result in Section 2.

Remark. We have already observed that, for $h \equiv 0, u \equiv 0$ is a solution of (1.2) for all $\lambda$. It follows from Lemma 4.1 that it is a strict local minimum of $\Phi_{\lambda, 0}$ for all $\lambda<\gamma(0)+\gamma(\infty) \lambda_{1}$. For $\lambda<\gamma(\infty)\left[1+\lambda_{1}\right], \mu \equiv \min \left\{0, \frac{\gamma(\infty)-\lambda}{\lambda_{1}}\right\}+\gamma(\infty)>0$ and for all $u \in X$,

$$
\Phi_{\lambda, 0}(u) \geq \frac{1}{2} \int_{\Omega}[\gamma(\infty)-\lambda] u^{2}+\gamma(\infty)|\nabla u|^{2} d x \geq \frac{\mu}{2}\|u\|^{2}
$$


showing that 0 is a strict global minimum of $\Phi_{\lambda, 0}$ in this case. In fact, for $\lambda<$ $\gamma(\infty)\left[1+\lambda_{1}\right], 0$ is the only solution of (1.2) for $h \equiv 0$ because $\Phi_{\lambda, 0}^{\prime}(u)=0$ implies

$$
0=\Phi_{\lambda, 0}^{\prime}(u) u \geq \int \gamma(\infty)\left[u^{2}+|\nabla u|^{2}\right]-\lambda u^{2} d x \geq \mu\|u\|^{2}
$$

by Proposition 3.1.

\section{Cerami sequences with localization}

Here we recall a result from [10] concerning the existence of special sequences of approximate critical points of a functional having what is usually known as a mountain pass geometry. In this section, $(X,\|\cdot\|)$ denotes any real Banach space and its dual space is denoted by $\left(X^{*},\|\cdot\|_{*}\right)$.

Proposition 2.1. Let $\Phi \in C^{1}(X, \mathbb{R})$ and $e \in X \backslash\{0\}$. Set $P(e)=\{p \in C([0,1], X)$ : $p(0)=0$ and $p(1)=e\}$ and then $c(e)=\inf _{p \in P(e)} \max _{t \in[0,1]} \Phi(p(t))$. Suppose that

$$
\max \{\Phi(0), \Phi(e)\}<c(e)
$$

and let $\left\{p_{n}\right\} \subset P(e)$ be a sequence of paths such that $M_{n}=\max _{t \in[0,1]} \Phi\left(p_{n}(t)\right) \rightarrow$ $c(e)$. Then there exists a sequence $\left\{u_{n}\right\} \subset X$ such that

$$
\Phi\left(u_{n}\right) \rightarrow c(e),\left(1+\left\|u_{n}\right\|\right)\left\|\Phi^{\prime}\left(u_{n}\right)\right\|_{*} \rightarrow 0 \text { and } \frac{d\left(u_{n}, p_{n}([0,1])\right)}{\left(1+\left\|u_{n}\right\|\right)} \rightarrow 0 \text { as } n \rightarrow \infty .
$$

Hence, if $S$ is a subset of $X$ such that $p_{n}([0,1]) \subset S$ for all $n$, then $\frac{d\left(u_{n}, S\right)}{1+\left\|u_{n}\right\|} \rightarrow 0$.

This result was proved in [10] as Corollary 1.2. Subsequently, P.J. Rabier [8] showed how it can be deduced from the much earlier work of Ghoussoub [6].

Remarks. (1) A functional $\Phi$ satisfying the condition (MPG) is said to have a mountain pass geometry since the mountain pass theorem then shows that $c(e)$ is a critical level of $\Phi$ provided that the Palais-Smale condition is also satisfied at this level $[1,6]$.

(2) A sequence having the properties $\Phi\left(u_{n}\right) \rightarrow c(e)$ and $(1+\|u\|)\left\|\Phi^{\prime}\left(u_{n}\right)\right\|_{*} \rightarrow 0$ is usually called a Cerami sequence for the level $c(e)$ because she seems to have been first to exploit weights of this kind in critical point theory, [3, 4]. See also [5].

(3) The property $d\left(u_{n}, S\right) /\left(1+\left\|u_{n}\right\|\right) \rightarrow 0$ gives some, albeit limited, information about the location of the sequence $\left\{u_{n}\right\}$ which may be helpful in proving that it is bounded. If one is ultimately able to prove the convergence of this special Cerami sequence, then one obtains a critical point in $S$. An example in [10] shows that, under our hypotheses, there may be no Cerami sequence for which $d\left(u_{n}, S\right)$ is bounded as $n \rightarrow \infty$.

(4) Suppose that $\left\|u_{n}\right\| \rightarrow \infty$. In the case where $p_{n}([0,1]) \subset S$ for all $n \in \mathbb{N}$ and $S=t S$ for all $t>0$, the information that $\frac{d\left(u_{n}, S\right)}{1+\left\|u_{n}\right\|} \rightarrow 0$ can be exploited in the 
following way. Let $K>0$ be fixed and set $z_{n}=t_{n} u_{n}$ where $t_{n}=K /\left\|u_{n}\right\|$. Then, for $u_{n} \neq 0$,

$$
\begin{array}{r}
d\left(u_{n}, S\right)=\inf _{v \in S}\left\|u_{n}-v\right\|=\frac{1}{t_{n}} \inf _{v \in S}\left\|z_{n}-t_{n} v\right\| \\
=\frac{1}{t_{n}} \inf _{z \in t_{n} S=S}\left\|z_{n}-z\right\|=\frac{\left\|u_{n}\right\|}{K} d\left(z_{n}, S\right) .
\end{array}
$$

and so

$$
d\left(z_{n}, S\right)=\frac{K\left(1+\left\|u_{n}\right\|\right)}{\left\|u_{n}\right\|} \frac{d\left(u_{n}, S\right)}{1+\left\|u_{n}\right\|} \rightarrow 0 .
$$

If in addition, $X$ is reflexive and $S$ is a cone (i.e. a closed convex subset of $X$ such that $t S=S$ for all $t>0$ ), we can go further by passing to a subsequence such that $z_{n} \rightarrow z$ weakly in $X$. Since $d\left(z_{n}, S\right) \rightarrow 0$, there exist $s_{n} \in S$ and $r_{n} \in X$ such that $z_{n}=s_{n}+r_{n}$ and $\left\|r_{n}\right\| \rightarrow 0$. This implies that $s_{n} \rightarrow z$ and, since $S$ is closed and convex, we conclude that $z \in S$.

\section{Basic properties of $\Phi_{\lambda, h}$}

The condition (g1) implies that $\Gamma$ is concave and $\gamma(\infty) t \leq \Gamma(t) \leq \gamma(0) t$ for all $t \geq 0$. In particular,

$$
\int_{\Omega} \Gamma\left(\frac{1}{2}\left[u^{2}+|\nabla u|^{2}\right]\right) d x \leq \frac{\gamma(0)}{2} \int_{\Omega} u^{2}+|\nabla u|^{2} d x
$$

and, for any $\lambda \in \mathbb{R}$, we can define a functional $\Phi_{\lambda, h}: X=H_{0}^{1}(\Omega) \rightarrow \mathbb{R}$ by (1.1). Furthermore, since $\Gamma \in C^{1}([0, \infty))$ with $\Gamma(0)=0$ we have that

$$
\Gamma(t)=\Gamma^{\prime}(0) t-G(t) t \text { where } G \in C^{1}((0, \infty)) \text { with } \lim _{t \rightarrow 0} G(t)=0 .
$$

Setting $G(0)=0$ we have $G \in C([0, \infty))$ and $0 \leq G(t) \leq \gamma(0)-\gamma(\infty)$.

Proposition 3.1. (I) If $\Gamma$ satisfies (g1), then $\Phi_{\lambda, h} \in C^{1}(X, \mathbb{R})$ for all $\lambda \in \mathbb{R}$ with

$$
\Phi_{\lambda, h}^{\prime}(u) v=\int_{\Omega} \gamma\left(\frac{1}{2}\left[u^{2}+|\nabla u|^{2}\right]\right)[u v+\nabla u \cdot \nabla v]-\lambda u v-h v d x
$$

for all $u, v \in X$.

(II) If in addition (g2) is satisfied, $\Phi_{\lambda, h}: X \rightarrow \mathbb{R}$ is weakly sequentially lower semicontinuous.

Proof. (I) Defining $F: \Omega \times \mathbb{R} \times \mathbb{R}^{N} \rightarrow \mathbb{R}$ by $F(x, s, p)=\Gamma\left(\frac{1}{2}\left[s^{2}+|p|^{2}\right]\right)-\frac{\lambda}{2} s^{2}-s h(x)$ one easily checks that the hypotheses of C.1 Theorem in [9] are satisfied.

(II) Let $w_{n} \rightarrow w$ weakly in $X$. Then $\int_{\Omega}\left(w_{n}-w\right) h d x \rightarrow 0$ and, by the compactness of the Sobolev embedding, $\int_{\Omega}\left(w_{n}-w\right)^{2} d x \rightarrow 0$. Hence it is enough to prove that

$$
\Phi_{0,0}(w) \leq \liminf _{n \rightarrow \infty} \Phi_{0,0}\left(w_{n}\right)
$$


For $u, v \in X$, let $y=(u, \nabla u)$ and $z=(v, \nabla v)$ and so that $y \cdot z=u v+\nabla u \cdot \nabla v$. Then, using (g2),

$$
\begin{aligned}
& \Phi_{0,0}(u)-\Phi_{0,0}(v)=\int_{\Omega} g\left(\frac{|y|}{\sqrt{2}}\right)-g\left(\frac{|z|}{\sqrt{2}}\right) d x \\
& \geq \int_{\Omega} g^{\prime}\left(\frac{|z|}{\sqrt{2}}\right)\left\{\frac{|y|-|z|}{\sqrt{2}}\right\} d x=\int_{\Omega} \gamma\left(\frac{|z|^{2}}{2}\right) \sqrt{2}|z|\left\{\frac{|y|-|z|}{\sqrt{2}}\right\} d x .
\end{aligned}
$$

From part(I) we also have that

$$
\begin{aligned}
& \Phi_{0,0}^{\prime}(v)(u-v)=\int_{\Omega} \gamma\left(\frac{|z|^{2}}{2}\right)\{v(u-v)+\nabla v \cdot \nabla(u-v)\} d x \\
& =\int_{\Omega} \gamma\left(\frac{|z|^{2}}{2}\right) z \cdot(y-z) d x .
\end{aligned}
$$

Hence we obtain

$$
\begin{aligned}
& \Phi_{0,0}(u)-\Phi_{0,0}(v)-\Phi_{0,0}^{\prime}(v)(u-v) \geq \int_{\Omega} \gamma\left(\frac{|z|^{2}}{2}\right)\{|z|(|y|-|z|)-z \cdot(y-z)\} \\
& =\int_{\Omega} \gamma\left(\frac{|z|^{2}}{2}\right)\{|z||y|-z \cdot y\} d x \geq 0 .
\end{aligned}
$$

Putting $u=w_{n}$ and $v=w$, this yields

$$
\Phi_{0,0}(w) \leq \Phi_{0,0}\left(w_{n}\right)-\Phi_{0,0}^{\prime}(w)\left(w_{n}-w\right),
$$

where $\Phi_{0,0}^{\prime}(w)\left(w_{n}-w\right) \rightarrow 0$ since $\Phi_{0,0}^{\prime}(w) \in X^{*}$ and $w_{n} \rightarrow w$ weakly in $X$.

Remark. For any $u \in X$, we have that $\Phi_{\lambda, h}^{\prime}(u)=0 \Leftrightarrow$

$$
\int_{\Omega} \gamma\left(\frac{1}{2}\left[u^{2}+|\nabla u|^{2}\right]\right)[u v+\nabla u \cdot \nabla v]-\lambda u v-h v d x=0 \text { for all } v \in X
$$

and this precisely what is meant by a weak solution of (1.2).

\section{Existence of a local minimum}

Lemma 4.1. Let (g1) be satisfied and consider $\lambda<\gamma(0)+\gamma(\infty) \lambda_{1}$. Set $\mu_{\lambda}=$ $\min \left\{\gamma(\infty), \gamma(\infty)+\frac{\gamma(0)-\lambda}{\lambda_{1}}\right\}$.

(I) Then $\mu_{\lambda}>0$ and there exists $R_{\lambda}>0$ such that $\Phi_{\lambda, 0}(u) \geq \frac{\mu_{\lambda}}{4}\|u\|^{2}$ for all $u \in X$ with $\|u\| \leq R_{\lambda}$.

(II) For $\|u\|=R_{\lambda}$ and $|h|_{2} \leq \frac{\mu_{\lambda}}{8} R_{\lambda} \sqrt{\lambda_{1}} \equiv H_{\lambda}$, we also have $\Phi_{\lambda, h}(u) \geq \frac{\mu_{\lambda}}{8} R_{\lambda}^{2} \equiv \varepsilon_{\lambda}$.

Proof. For all $u \in X$, the concavity of $\Gamma$ implies that

$$
\Gamma\left(\frac{1}{2}\left[u^{2}+|\nabla u|^{2}\right]\right) \geq \frac{1}{2} \Gamma\left(u^{2}\right)+\frac{1}{2} \Gamma\left(|\nabla u|^{2}\right) \geq \frac{1}{2}\left[\gamma(0) u^{2}-G\left(u^{2}\right) u^{2}\right]+\frac{1}{2} \gamma(\infty)|\nabla u|^{2},
$$


where $G$ is defined by (3.1). Also, for $1<p<2^{*} / 2$ we have that

$$
\begin{aligned}
0 & \leq \int_{\Omega} G\left(u^{2}\right) u^{2} d x \leq\left\{\int_{\Omega} G\left(u^{2}\right)^{p^{\prime}} d x\right\}^{\frac{1}{p^{\prime}}}\left\{\int_{\Omega} u^{2 p} d x\right\}^{\frac{1}{p}} \\
& \leq\left\{\int_{\Omega} G\left(u^{2}\right)^{p^{\prime}} d x\right\}^{\frac{1}{p^{\prime}}} C_{2 p}^{2}\|u\|^{2}
\end{aligned}
$$

where $2^{*}$ and $C_{2 p}$ are the critical Sobolev exponent and constant for the embedding of $X$ in $L^{2 p}(\Omega)$. Furthermore,

$$
\begin{aligned}
& {[\gamma(0)-\lambda] \int_{\Omega} u^{2} d x \geq 0 \text { if } \lambda \leq \gamma(0) \text { and }} \\
& {[\gamma(0)-\lambda] \int_{\Omega} u^{2} d x \geq \frac{\gamma(0)-\lambda}{\lambda_{1}} \int_{\Omega}|\nabla u|^{2} d x \text { if } \lambda>\gamma(0)}
\end{aligned}
$$

Hence

$$
\int_{\Omega} \gamma(\infty)|\nabla u|^{2}+[\gamma(0)-\lambda] u^{2} d x \geq \mu_{\lambda} \int_{\Omega}|\nabla u|^{2} d x
$$

and so

$$
\Phi_{\lambda, 0}(u) \geq \frac{\mu_{\lambda}}{2}\|u\|^{2}-\frac{1}{2}\left\{\int_{\Omega} G\left(u^{2}\right)^{p^{\prime}} d x\right\}^{\frac{1}{p^{\prime}}} C_{2 p}^{2}\|u\|^{2} \text { for all } u \in X .
$$

But $0 \leq G(t) \leq \gamma(0)-\gamma(\infty)$ for all $t \in \mathbb{R}$ and so $u \mapsto G\left(u^{2}\right)^{p^{\prime}}$ is continuous from $L^{2}(\Omega)$ into $L^{1}(\Omega)$. Since $G(0)=0$, it follows that there exists $\delta_{\lambda}>0$ such that $\left\{\int_{\Omega} G\left(u^{2}\right)^{p^{\prime}} d x\right\}^{\frac{1}{p^{\prime}}} \leq \frac{\mu_{\lambda}}{2 C_{2 p}^{2}}$ for $u \in L^{2}(\Omega)$ with $|u|_{2} \leq \delta_{\lambda}$. Therefore, setting $R_{\lambda}=\sqrt{\lambda_{1}} \delta_{\lambda}$,

$$
\Phi_{\lambda, 0}(u) \geq \frac{\mu_{\lambda}}{4}\|u\|^{2} \text { for } u \in X \text { with }\|u\| \leq R_{\lambda} .
$$

(II) For all $u \in X,|u|_{2}^{2} \leq\|u\|^{2} / \lambda_{1}$ and $\left|\int_{\Omega} u h d x\right| \leq|u|_{2}|h|_{2}$, so it follows from part (I) that

$$
\Phi_{\lambda, h}(u) \geq \frac{\mu_{\lambda}}{4}\|u\|^{2}-\frac{1}{\sqrt{\lambda}_{1}}\|u\||h|_{2}=\|u\|\left\{\frac{\mu_{\lambda}}{4}\|u\|-\frac{1}{\sqrt{\lambda}_{1}}|h|_{2}\right\} \geq \frac{\mu_{\lambda}}{8} R_{\lambda}^{2}
$$

for $\|u\|=R_{\lambda}$ and $|h|_{2} \leq \frac{\mu_{\lambda}}{8} R_{\lambda} \sqrt{\lambda_{1}}$.

In the notation of the lemma, we now consider the open ball, $B\left(0, R_{\lambda}\right)$ in $X$ with centre 0 and radius $R_{\lambda}$. Its closure and boundary are denoted by $\overline{B\left(0, R_{\lambda}\right)}$ and $\partial B\left(0, R_{\lambda}\right)$, respectively.

Theorem 4.2. Let (g1) and (g2) be satisfied with $\lambda<\gamma(0)+\gamma(\infty) \lambda_{1}$ and $|h|_{2} \leq H_{\lambda}$, where $H_{\lambda}$ and $R_{\lambda}$ are given by Lemma 4.1. There exists $u_{1}=u_{1}(\lambda, h) \in B\left(0, R_{\lambda}\right)$ such that $\Phi_{\lambda, h}\left(u_{1}\right) \leq \Phi_{\lambda, h}(v)$ for all $v \in \overline{B\left(0, R_{\lambda}\right)}$. Furthermore, $u_{1} \geq 0$ on $\Omega$ and $\Phi_{\lambda, h}^{\prime}\left(u_{1}\right)=0$. Also, $\Phi_{\lambda, h}\left(u_{1}\right)<0$ if $h \not \equiv 0$ whereas $u_{1} \equiv 0$ for $h \equiv 0$.

Proof. With $\lambda$ and $h$ fixed, let $m=\inf \left\{\Phi_{\lambda, h}(u): u \in \overline{B\left(0, R_{\lambda}\right)}\right\}$ and consider a sequence $\left\{v_{n}\right\} \subset \overline{B\left(0, R_{\lambda}\right)}$ such that $\Phi_{\lambda, h}\left(v_{n}\right) \rightarrow m$. By passing to a subsequence, we may suppose that $v_{n} \rightarrow v$ weakly in $X$ for some element $v \in \overline{B\left(0, R_{\lambda}\right)}$ since $X$ is reflexive and $\overline{B\left(0, R_{\lambda}\right)}$ is closed and convex. By Proposition 3.1(II), $\Phi_{\lambda, h}(v)=m$ 
and by Lemma $4.1, m \leq \Phi_{\lambda, h}(0)=0<\varepsilon_{\lambda} \leq \inf \left\{\Phi_{\lambda, h}(u): u \in \partial B\left(0, R_{\lambda}\right)\right\}$, showing that $v \in B\left(0, R_{\lambda}\right)$.

Set $u_{1}=|v|$. Recalling that, for all $u \in X,|u| \in X$ and $|\nabla u|=|\nabla| u||$ a.e. on $\Omega$, we have that $u_{1} \in X$ with $\left\|u_{1}\right\|=\|v\|$ and $\Phi_{\lambda, 0}\left(u_{1}\right)=\Phi_{\lambda, 0}(v)$. Since $h \geq 0$ a.e. on $\Omega$, we have that $\int_{\Omega} v h d x \leq \int_{\Omega} u_{1} h d x$ and so $\Phi_{\lambda, h}\left(u_{1}\right) \leq \Phi_{\lambda, h}(v)=m$, too. Thus $u_{1} \in B\left(0, R_{\lambda}\right)$ and $\Phi_{\lambda, h}\left(u_{1}\right)=m \leq 0$. It follows from Proposition 3.1(I) that $\Phi_{\lambda, h}^{\prime}\left(u_{1}\right)=0$. For $h \equiv 0$, Lemma $4.1(\mathrm{I})$ shows that $u_{1}=0$ and hence $\Phi_{\lambda, h}\left(u_{1}\right)=0$. If $h \not \equiv 0$ and $\Phi_{\lambda, h}\left(u_{1}\right)=0$, we have that $m=0=\Phi_{\lambda, h}(0)$ and so $\Phi_{\lambda, h}^{\prime}(0)=0$. But this means that $u \equiv 0$ satisfies (1.2) which implies that $h \equiv 0$. Hence $\Phi_{\lambda, h}\left(u_{1}\right)<0$ when $h \neq \equiv 0$.

\section{The mountain pass geometry of $\Phi_{\lambda, h}$}

Lemma 5.1. Suppose that $\Gamma$ satisfies (g1). For all $\lambda \in \mathbb{R}$,

$$
\frac{\Phi_{\lambda, h}(t \phi)}{t^{2}} \rightarrow \frac{\gamma(\infty)\left[1+\lambda_{1}\right]-\lambda}{2} \int_{\Omega} \phi(x)^{2} d x \text { as } t \rightarrow \infty
$$

where $\phi$ is the positive eigenfunction associated with $\lambda_{1}$.

Proof. Since $\phi \in C^{1}(\Omega)$ and $\phi(x)>0$ for all $x \in \Omega$, we have that

$$
\frac{\Gamma\left(\frac{t^{2}}{2}\left[\phi(x)^{2}+|\nabla \phi(x)|^{2}\right]\right)}{t^{2}} \rightarrow \frac{1}{2} \gamma(\infty)\left[\phi(x)^{2}+|\nabla \phi(x)|^{2}\right] \text { as } t \rightarrow \infty
$$

for all $x \in \Omega$. On the other hand,

$$
0 \leq \frac{\Gamma\left(\frac{t^{2}}{2}\left[\phi(x)^{2}+|\nabla \phi(x)|^{2}\right]\right)}{t^{2}} \leq \frac{1}{2} \gamma(0)\left[\phi(x)^{2}+|\nabla \phi(x)|^{2}\right]
$$

for all $x \in \Omega$ and $t \neq 0$ where $\phi \in H_{0}^{1}(\Omega)$. As $t \rightarrow \infty$, the Dominated Convergence Theorem yields

$$
\begin{aligned}
\frac{\Phi_{\lambda, h}(t \phi)}{t^{2}} & =\int_{\Omega} \frac{\Gamma\left(\frac{t^{2}}{2}\left[\phi(x)^{2}+|\nabla \phi(x)|^{2}\right]\right)}{t^{2}}-\frac{1}{2} \lambda \phi(x)^{2}-\frac{\phi(x) h(x)}{t} d x \\
& \rightarrow \frac{1}{2} \int_{\Omega}[\gamma(\infty)-\lambda] \phi(x)^{2}+\gamma(\infty)|\nabla \phi(x)|^{2} d x \\
& =\frac{1}{2} \int_{\Omega}\left[\gamma(\infty)-\lambda+\gamma(\infty) \lambda_{1}\right] \phi(x)^{2} d x .
\end{aligned}
$$

Combining Lemmas 4.1 and 5.1 we see that $\Phi_{\lambda, h}$ has a mountain pass geometry for some values of $\lambda$. We use the notation introduced in Section 2 .

Corollary 5.2. Suppose that (g1) holds and consider $(\lambda, h)$ such that

$$
\gamma(\infty)+\gamma(\infty) \lambda_{1}<\lambda<\gamma(0)+\gamma(\infty) \lambda_{1} \quad \text { and } \quad|h|_{2} \leq H_{\lambda} .
$$

Then $\Phi_{\lambda, h}$ satisfies $(M P G)$ with $c(e)>0$ for $e=T \phi$, where $T>0$ is large enough so that $\Phi_{\lambda, h}(T \phi)<0$. 
Proof. By Lemma 4.1(II), there exists $R_{\lambda}>0$ such that $\inf _{\|u\|=R_{\lambda}} \Phi_{\lambda, h}(u) \geq \varepsilon_{\lambda}>0$. Since $\lambda>\gamma(\infty)\left[1+\lambda_{1}\right]$ it follows from Lemma 5.1 that we can choose $T>0$ so large that $T\|\phi\|>R_{\lambda}$ and $\Phi_{\lambda, h}(T \phi)<0$. Then $\max \left\{\Phi_{\lambda, h}(0), \Phi_{\lambda, h}(T \phi)\right\} \leq 0$. But for any $p \in P(T \phi)$ there exists $t \in(0,1)$ such that $\|p(t)\|=R_{\lambda}$ and so $\max _{t \in[0,1]} \Phi_{\lambda, h}(p(t)) \geq$ $\varepsilon_{\lambda}$, showing that (MPG) is satisfied with $c(T \phi) \geq \varepsilon_{\lambda}$.

\section{Existence of a special Cerami sequence for $\Phi_{\lambda, h}$}

Using Proposition 2.1 we obtain a Cerami sequence for $\Phi_{\lambda, h}$ having some additional properties which will enable us to show that it has a convergent subsequence.

Proposition 6.1. Let (g1) be satisfied and consider $(\lambda, h)$ such that $\gamma(\infty)\left[1+\lambda_{1}\right]<$ $\lambda<\gamma(0)+\gamma(\infty) \lambda_{1}$ and $|h|_{2} \leq H_{\lambda}$. Let $S=\{u \in X: u \geq 0$ a.e. in $\Omega\}$. Then there exists a sequence $\left\{u_{n}\right\} \subset X$ such that

$$
\Phi_{\lambda, h}\left(u_{n}\right) \rightarrow c>0,\left(1+\left\|u_{n}\right\|\right) \Phi_{\lambda, h}^{\prime}\left(u_{n}\right) \rightarrow 0 \text { and } \frac{d\left(u_{n}, S\right)}{1+\left\|u_{n}\right\|} \rightarrow 0 .
$$

Furthermore, one of the following cases occurs: either (i) $u_{n} \rightarrow u \in S$ weakly in $X$ or (ii) $\left\|u_{n}\right\| \rightarrow \infty$. In case (ii), for any $K>0$, the sequence can be chosen so that $w_{n}=K \frac{u_{n}}{\left\|u_{n}\right\|} \rightarrow w \in S$ weakly in $X$.

Proof. By Proposition 3.1(I) and Corollary 5.2, the hypotheses of Proposition 2.1 are satisfied by $\Phi_{\lambda, h}$ with $c=c(e)>0$ and $e=T \phi$ for $T>0$ sufficiently large. Let $\left\{p_{n}\right\} \subset P(e)$ be a sequence of paths such that $M_{n} \rightarrow c$. Since $e \in S$ and $u \mapsto|u|$ is a continuous mapping from $X$ into itself (see Proposition 5.1 in [10], $\left|p_{n}\right| \in P(e)$ for all $n$ and $\left|p_{n}\right|(t)=\left|p_{n}(t)\right| \in S$ for all $n \in \mathbb{N}$ and $t \in[0,1]$. But, for all $u \in X,|\nabla u|=|\nabla| u||$ a.e. on $\Omega$ and so $\Phi_{\lambda, 0}(u)=\Phi_{\lambda, 0}(|u|)$ for all $u \in X$. Since $h \geq 0$ a.e. on $\Omega$, we also have that $\int_{\Omega} u h d x \leq \int_{\Omega}|u| h d x$ for all $u \in X$ and so $\Phi_{\lambda, h}(|u|) \leq \Phi_{\lambda, h}(u)$, too. Hence $\Phi_{\lambda, h}\left(\left|p_{n}\right|(t)\right) \leq \Phi_{\lambda, h}\left(p_{n}(t)\right)$ for all $t \in[0,1]$ and $\max _{t \in[0,1]} \Phi_{\lambda, h}\left(\left|p_{n}\right|(t)\right)=M_{n} \rightarrow c$. It follows from Proposition 2.1 that there exists a sequence $\left\{u_{n}\right\} \in X$ satisfying (6.1) with $\Phi=\Phi_{\lambda, h}$.

If $\left\|u_{n}\right\| \not \rightarrow \infty$, by passing to a subsequence, we can suppose that $u_{n} \rightarrow u$ weakly in $X$ and $d\left(u_{n}, S\right) \rightarrow 0$. Then there exists a sequence $\left\{v_{n}\right\} \subset S$ such that $\left\|u_{n}-v_{n}\right\| \rightarrow 0$ and consequently, $v_{n} \rightarrow u$ weakly in $X$. Since $S$ is closed and convex, this implies that $u \in S$. Thus we obtain case (i) whenever case (ii) does not occur. In case (ii), the Remark 4 following Proposition 2.1 shows that the subsequence can be chosen so that $w_{n}=K \frac{u_{n}}{\left\|u_{n}\right\|} \rightarrow w \in S$.

\section{The special Cerami sequence is bounded in $X$}

The next step is to show that case (ii) in Proposition 6.1 cannot occur and for this the following properies of $\Phi_{\lambda, h}$ will be useful. 
Lemma 7.1. (a) If (g1) is satisfied, then

$$
\Phi_{\lambda, 0}(t u) \leq \Phi_{\lambda, 0}(u)+\frac{t^{2}-1}{2} \Phi_{\lambda, 0}^{\prime}(u) u \text { for all } \lambda, t \in \mathbb{R} \text { and } u \in X .
$$

(b) If in addition (g3) holds, then

$$
\Phi_{\lambda, h}(t u) \leq t(2-t) \Phi_{\lambda, h}(u)+t(t-1) \Phi_{\lambda, h}^{\prime}(u) u+(1-t)^{2} K(\infty)|\Omega|
$$

for all $\lambda, t \in \mathbb{R}, u \in X$ and $h \in L^{2}(\Omega)$, where $K(\infty)=\lim _{t \rightarrow \infty}[\Gamma(t)-\gamma(t) t]$.

Proof. (a) Fix $\lambda \in \mathbb{R}$ and $u \in X$. For $s \geq 0$, consider the function $q:[0, \infty) \rightarrow \mathbb{R}$ defined by

$$
q(s)=\Phi_{\lambda, 0}(\sqrt{s} u)=\int_{\Omega} \Gamma\left(\frac{s}{2}\left[u^{2}+|\nabla u|^{2}\right]\right)-\frac{\lambda}{2} s u^{2} d x .
$$

By Proposition 3.1, $q \in C^{1}((0, \infty)) \cap C([0, \infty))$ with $q^{\prime}(s)=\Phi_{\lambda, 0}^{\prime}(\sqrt{s} u) \frac{1}{2 \sqrt{s}} u$ for $s>0$. But, by $(\mathrm{g} 1), q$ is also concave on $[0, \infty)$ and so $q(s) \leq q(1)+q^{\prime}(1)(s-1)$ for all $s \geq 0$. Putting $s=t^{2}$ we get

$$
\Phi_{\lambda, 0}(t u)=\Phi_{\lambda, 0}(|t| u)=q(s) \leq \Phi_{\lambda, 0}(u)+\frac{t^{2}-1}{2} \Phi_{\lambda, 0}^{\prime}(u) u
$$

as required.

(b) Since $\Phi_{\lambda, h}(u)=\Phi_{\lambda, 0}(u)-\int_{\Omega} u h d x$ and $\Phi_{\lambda, h}^{\prime}(u) u=\Phi_{\lambda, 0}^{\prime}(u) u-\int_{\Omega} u h d x$, it follows from part (a) that

$$
\Phi_{\lambda, h}(t u)+t \int_{\Omega} u h d x \leq \Phi_{\lambda, h}(u)+\int_{\Omega} u h d x+\frac{t^{2}-1}{2}\left\{\Phi_{\lambda, h}^{\prime}(u) u+\int_{\Omega} u h d x\right\}
$$

and hence

$$
\Phi_{\lambda, h}(t u) \leq \Phi_{\lambda, h}(u)+\frac{t^{2}-1}{2} \Phi_{\lambda, h}^{\prime}(u) u+\frac{(1-t)^{2}}{2} \int_{\Omega} u h d x
$$

for all $\lambda, t \in \mathbb{R}$ and $u \in X$. But

$$
2 \Phi_{\lambda, h}(u)-\Phi_{\lambda, h}^{\prime}(u) u=\int_{\Omega} 2 K\left(\frac{1}{2}\left[u^{2}+|\nabla u|^{2}\right]\right)-u h d x
$$

where $K(t)=\Gamma(t)-\gamma(t) t$ is non-decreasing and so

$$
\int_{\Omega} u h d x \leq 2 K(\infty)|\Omega|-2 \Phi_{\lambda, h}(u)+\Phi_{\lambda, h}^{\prime}(u) u,
$$

proving part (b).

Lemma 7.2. Suppose that (g1) is satisfied. Let $\left\{w_{n}\right\}$ be a sequence in $X$ such that $w_{n} \rightarrow w$ weakly in $X$ and let $\left\{t_{n}\right\} \subset \mathbb{R} \backslash\{0\}$ be a sequence such that $t_{n} \rightarrow 0$ and $t_{n} \Phi_{\lambda, h}^{\prime}\left(\frac{w_{n}}{t_{n}}\right) \rightarrow 0$ in $X^{*}$. Then

$$
\int_{\Omega} \gamma(\infty)[w v+\nabla w \cdot \nabla v]-\lambda w v d x=0 \text { for all } v \in X
$$


Proof. By (3.2) we have

$$
t_{n} \Phi_{\lambda, h}^{\prime}\left(\frac{w_{n}}{t_{n}}\right) v=\int_{\Omega} \gamma\left(\frac{1}{2 t_{n}^{2}}\left[w_{n}^{2}+\left|\nabla w_{n}\right|^{2}\right]\right)\left[w_{n} v+\nabla w_{n} \cdot \nabla v\right]-\lambda w_{n} v-t_{n} v h d x
$$

and so

$$
\int_{\Omega} \gamma\left(\frac{1}{2 t_{n}^{2}}\left[w_{n}^{2}+\left|\nabla w_{n}\right|^{2}\right]\right)\left[w_{n} v+\nabla w_{n} \cdot \nabla v\right]-\lambda w_{n} v d x \rightarrow 0
$$

Since

$$
\int_{\Omega} w_{n} v d x \rightarrow \int_{\Omega} w v d x \text { and } \int_{\Omega}\left[w_{n} v+\nabla w_{n} \cdot \nabla v\right] d x \rightarrow \int_{\Omega}[w v+\nabla w \cdot \nabla v] d x,
$$

it is enough to prove that

$$
\int_{\Omega}\left\{\gamma\left(\frac{1}{2 t_{n}^{2}}\left[w_{n}^{2}+\left|\nabla w_{n}\right|^{2}\right]\right)-\gamma(\infty)\right\}\left[w_{n} v+\nabla w_{n} \cdot \nabla v\right] d x \rightarrow 0 .
$$

Let $z_{n}=\left(w_{n}, \nabla w_{n}\right)$ and $y=(v, \nabla v)$. Then $z_{n}, y \in\left[L^{2}(\Omega)\right]^{N+1}$ and there exists a constant $Z$ such that $\int_{\Omega}\left|z_{n}\right|^{2} d x \leq Z$ for all $n$ since $\left\{w_{n}\right\}$ is bounded in $X$. Also

$$
\begin{aligned}
& \int_{\Omega}\left\{\gamma\left(\frac{1}{2 t_{n}^{2}}\left[w_{n}^{2}+\left|\nabla w_{n}\right|^{2}\right]\right)-\gamma(\infty)\right\}\left[w_{n} v+\nabla w_{n} \cdot \nabla v\right] d x \\
& =\int_{\Omega}\left\{\gamma\left(\frac{1}{2 t_{n}^{2}}\left|z_{n}\right|^{2}\right)-\gamma(\infty)\right\} z_{n} \cdot y d x \\
& =\left(\int_{A_{n}^{m}}+\int_{B_{n}^{m}}\right)\left\{\gamma\left(\frac{1}{2 t_{n}^{2}}\left|z_{n}\right|^{2}\right)-\gamma(\infty)\right\} z_{n} \cdot y d x
\end{aligned}
$$

where $A_{n}^{m}=\left\{x \in \Omega:\left|z_{n}\right|^{2} \leq 1 / m\right\}$ and $B_{n}^{m}=\Omega \backslash A_{n}^{m}$ for $m, n \in \mathbb{N}$. For all $m, n \in \mathbb{N}$,

$$
\begin{aligned}
& \int_{A_{n}^{m}}\left|\gamma\left(\frac{1}{2 t_{n}^{2}}\left|z_{n}\right|^{2}\right)-\gamma(\infty)\right|\left|z_{n}\right||y| d x \leq \frac{\gamma(0)-\gamma(\infty)}{\sqrt{m}} \int_{A_{n}^{m}}|y| d x \\
& \leq \frac{\gamma(0)-\gamma(\infty)}{\sqrt{m}}|\Omega|^{1 / 2}\left\{\int_{\Omega}|y|^{2} d x\right\}^{1 / 2} .
\end{aligned}
$$

But, for every $m \in \mathbb{N}$, there exists $S_{m}>0$ such that $|\gamma(s)-\gamma(\infty)|<1 / m$ for all $s \geq S_{m}$. Since $t_{n} \rightarrow 0$, there exists $N(m)>0$ such that $t_{n}^{2} \leq \frac{1}{2 m S_{m}}$ for all $n \geq N(m)$. Hence, on $B_{n}^{m},\left|\gamma\left(\frac{1}{2 t_{n}^{2}}\left|z_{n}\right|^{2}\right)-\gamma(\infty)\right|<1 / m$ for $n \geq N(m)$ and so

$$
\begin{aligned}
& \int_{B_{n}^{m}}\left|\gamma\left(\frac{1}{2 t_{n}^{2}}\left|z_{n}\right|^{2}\right)-\gamma(\infty)\right|\left|z_{n}\right||y| d x \leq \frac{1}{m}\left\{\int_{\Omega}\left|z_{n}\right|^{2} d x\right\}^{1 / 2}\left\{\int_{\Omega}|y|^{2} d x\right\}^{1 / 2} \\
& \leq \frac{Z^{1 / 2}}{m}\left\{\int_{\Omega}|y|^{2} d x\right\}^{1 / 2}
\end{aligned}
$$

for all $n \geq N(m)$. Thus

$$
\int_{\Omega}\left|\gamma\left(\frac{1}{2 t_{n}^{2}}\left|z_{n}\right|^{2}\right)-\gamma(\infty)\right|\left|z_{n}\right||y| d x \leq\left\{\frac{\gamma(0)-\gamma(\infty)}{\sqrt{m}}|\Omega|^{1 / 2}+\frac{Z^{1 / 2}}{m}\right\}\left\{\int_{\Omega}|y|^{2} d x\right\}^{1 / 2}
$$

for all $m \in \mathbb{N}$ and all $n \geq N(m)$. It follows that

$$
\int_{\Omega}\left|\gamma\left(\frac{1}{2 t_{n}^{2}}\left|z_{n}\right|^{2}\right)-\gamma(\infty)\right|\left|z_{n}\right||y| d x \rightarrow 0 \text { as } n \rightarrow \infty
$$


completing the proof.

Proposition 7.3. Under the hypotheses of Proposition 6.1, and with the additional assumption that (g3) holds when $h \not \equiv 0$, there exist a sequence $\left\{u_{n}\right\} \subset X$ and $u \in S$ such that $u_{n} \rightarrow u$ weakly in $X$ and (6.1) is satisfied.

Proof. We need only show that case (ii) of Proposotion 6.1 cannot occur. Arguing by contradiction, we suppose that there is a sequence $\left\{u_{n}\right\}$ satisfying $(6.1)$ such that $\left\|u_{n}\right\| \rightarrow \infty$. Set

$$
w_{n}=K \frac{u_{n}}{\left\|u_{n}\right\|} \text { where } K>0 \text { is a constant to be chosen later . }
$$

Then $t_{n}=K /\left\|u_{n}\right\| \rightarrow 0$ as $n \rightarrow \infty$ and, as noted in Proposition 6.1 , by passing to a subsequence we can assume that $w_{n} \rightarrow w$ weakly in $X$ where $w \in S$. Since $\Phi_{\lambda, h}\left(w_{n}\right)=\Phi_{\lambda, h}\left(t_{n} u_{n}\right)$ it follows from Lemma 7.1 that

$$
\limsup _{n \rightarrow \infty} \Phi_{\lambda, 0}\left(w_{n}\right) \leq c \text { and } \limsup _{n \rightarrow \infty} \Phi_{\lambda, h}\left(w_{n}\right) \leq K(\infty)|\Omega| \text { when } h \not \equiv 0 .
$$

On the other hand,

$$
\begin{aligned}
\Phi_{\lambda, h}\left(w_{n}\right) & =\int_{\Omega} \Gamma\left(\frac{1}{2}\left[w_{n}^{2}+\left|\nabla w_{n}\right|^{2}\right]\right)-\frac{\lambda}{2} w_{n}^{2}-w_{n} h d x \\
& \geq \frac{1}{2} \int_{\Omega}[\gamma(\infty)-\lambda] w_{n}^{2}+\gamma(\infty)\left|\nabla w_{n}\right|^{2} d x-\int_{\Omega} w_{n} h d x \\
& =\frac{\gamma(\infty)-\lambda}{2} \int_{\Omega} w_{n}^{2} d x+\frac{\gamma(\infty)}{2} K^{2}-\int_{\Omega} w_{n} h d x,
\end{aligned}
$$

since $\left\|w_{n}\right\|=K$ for all $n$. If $w=0$, we have that $\int_{\Omega} w_{n}^{2} d x \rightarrow 0$, by the compactness of the embedding of $X$ in $L^{2}(\Omega)$, and consequently

$$
\liminf _{n \rightarrow \infty} \Phi_{\lambda, h}\left(w_{n}\right) \geq \frac{\gamma(\infty)}{2} K^{2}
$$

But the constant $K>0$ is arbitrary and can be chosen so large that $\frac{\gamma(\infty)}{2} K^{2}>$ $c$, when $h \equiv 0$, and $\frac{\gamma(\infty)}{2} K^{2}>K(\infty)|\Omega|$, when $h \not \equiv 0$, contradicting our earlier conclusion about $\limsup _{n \rightarrow \infty} \Phi_{\lambda, h}\left(w_{n}\right)$.

Hence we have that $w \not \equiv 0$ and $w \geq 0$ since $w \in S$. Therefore $\int_{\Omega} w \phi d x>0$ where $\phi$ is the positive eigenfunction associated with $\lambda_{1}$. By Lemma 7.2, we have that

$$
\lambda \int_{\Omega} w \phi d x=\gamma(\infty) \int_{\Omega}[w \phi+\nabla w \cdot \nabla \phi] d x=\gamma(\infty) \int_{\Omega}\left[w \phi+\lambda_{1} w \phi\right] d x
$$

from which it follows that $\lambda=\gamma(\infty)\left[1+\lambda_{1}\right]$, contradicting our hypothesis that $\lambda>\gamma(\infty)\left[1+\lambda_{1}\right]$. Hence our assumption that $\left\|u_{n}\right\| \rightarrow \infty$ leads to a contradiction and the proof is complete. 


\section{Convergence of the special Cerami sequence}

Lemma 8.1. If (g1) and (g2) are satisfied, then

$$
\frac{\eta}{2}\|u-v\|^{2} \leq \Phi_{\lambda, h}(u)-\Phi_{\lambda, h}(v)+\left\|\Phi_{\lambda, h}^{\prime}(v)\right\|_{*}\|u-v\|+\frac{\lambda}{2}|u-v|_{2}^{2}
$$

for all $u, v \in X$ and all $\lambda \in \mathbb{R}$, where $\eta=\min \{\rho, \gamma(\infty)\}>0$.

Proof. For $u, v \in X$, set $w=(u, \nabla u)$ and $z=(v, \nabla v)$ so that $w \cdot z=u v+\nabla u \cdot \nabla v$. Then

$$
\begin{aligned}
& \Phi_{0,0}(u)-\Phi_{0,0}(v)=\int_{\Omega} g\left(\frac{|w|}{\sqrt{2}}\right)-g\left(\frac{|z|}{\sqrt{2}}\right) d x \\
& \geq \int_{\Omega} g^{\prime}\left(\frac{|z|}{\sqrt{2}}\right)\left\{\frac{|w|-|z|}{\sqrt{2}}\right\}+\frac{\rho}{2}(|w|-|z|)^{2} d x \\
& =\int_{\Omega} \gamma\left(\frac{|z|^{2}}{2}\right) \sqrt{2}|z|\left\{\frac{|w|-|z|}{\sqrt{2}}\right\}+\frac{\rho}{2}(|w|-|z|)^{2} d x \\
& \geq \int_{\Omega} \gamma\left(\frac{|z|^{2}}{2}\right)|z|(|w|-|z|)+\frac{\eta}{2}\left(|w-z|^{2}+2 w \cdot z-2|w||z|\right) d x
\end{aligned}
$$

We also have that

$$
\begin{aligned}
\Phi_{0,0}^{\prime}(v)(u-v) & =\int_{\Omega} \gamma\left(\frac{|z|^{2}}{2}\right)\{v(u-v)+\nabla v \cdot \nabla(u-v)\} d x \\
& =\int_{\Omega} \gamma\left(\frac{|z|^{2}}{2}\right) z \cdot(w-z) d x .
\end{aligned}
$$

Hence we obtain

$$
\begin{aligned}
& \Phi_{\lambda, h}(u)-\Phi_{\lambda, h}(v)-\Phi_{\lambda, h}^{\prime}(v)(u-v) \\
&=\Phi_{0,0}(u)-\Phi_{0,0}(v)-\Phi_{0,0}^{\prime}(v)(u-v)-\frac{\lambda}{2} \int_{\Omega} u^{2}-v^{2}-2 v(u-v) \\
& \geq \int_{\Omega} \gamma\left(\frac{|z|^{2}}{2}\right)\{|z|(|w|-|z|)-z \cdot(w-z)\}+\frac{\eta}{2}\left(|w-z|^{2}+2 w \cdot z-2|w||z|\right) \\
&-\frac{\lambda}{2}(u-v)^{2} d x \\
&= \int_{\Omega}\left\{\gamma\left(\frac{|z|^{2}}{2}\right)-\eta\right\}\{|z||w|-z \cdot w\}+\frac{\eta}{2}|w-z|^{2}-\frac{\lambda}{2}(u-v)^{2} d x \\
& \geq \int_{\Omega}\{\gamma(\infty)-\eta\}\{|z||w|-z \cdot w\}+\frac{\eta}{2}|w-z|^{2}-\frac{\lambda}{2}(u-v)^{2} d x \\
& \geq \int_{\Omega} \frac{\eta}{2}|w-z|^{2}-\frac{\lambda}{2}(u-v)^{2} d x .
\end{aligned}
$$

Hence

$$
\frac{\eta}{2} \int_{\Omega}|w-z|^{2} d x \leq \Phi_{\lambda, h}(u)-\Phi_{\lambda, h}(v)-\Phi_{\lambda, h}^{\prime}(v)(u-v)+\frac{\lambda}{2} \int_{\Omega}(u-v)^{2} d x .
$$


But $|w-z|^{2}=(u-v)^{2}+|\nabla(u-v)|^{2}$ and so we have

$$
\frac{\eta}{2}\|u-v\|^{2} \leq \Phi_{\lambda, h}(u)-\Phi_{\lambda, h}(v)-\Phi_{\lambda, h}^{\prime}(v)(u-v)+\frac{\lambda-\eta}{2} \int_{\Omega}(u-v)^{2} d x
$$

from which the desired conclusion follows easily.

Corollary 8.2. Under the hypotheses of Proposition 7.3, consider the special Cerami sequence $\left\{u_{n}\right\}$ for $\Phi_{\lambda, h}$ and its weak limit $u \in S$ given by that result. Suppose that $\Gamma$ satisfies (g2). Then $\left\|u_{n}-u\right\| \rightarrow 0$ and consequently, $\Phi_{\lambda, h}^{\prime}(u)=0$ with $\Phi_{\lambda, h}(u)=$ $c>0$.

Proof. By Lemma 8.1 we have

$$
\frac{\eta}{2}\left\|u_{n}-u_{m}\right\|^{2} \leq \Phi_{\lambda, h}\left(u_{n}\right)-\Phi_{\lambda, h}\left(u_{m}\right)+\left\|\Phi_{\lambda, h}^{\prime}\left(u_{m}\right)\right\|\left\|_{*}\right\| u_{n}-u_{m} \|+\frac{\lambda}{2}\left|u_{n}-u_{m}\right|_{2}^{2}
$$

for all $n, m$. Since $u_{n} \rightarrow u$ weakly in $X$, the compactness of the embedding into $L^{2}(\Omega)$ implies that $\left\{u_{n}\right\}$ is a Cauchy sequence in $L^{2}(\Omega)$. From (6.1) we have that $\lim _{m \rightarrow \infty}\left\|\Phi_{\lambda, h}^{\prime}\left(u_{m}\right)\right\|_{*}=0$. But the weak convergence of $\left\{u_{n}\right\}$ in $X$ also implies that $\left\{\left\|u_{n}\right\|\right\}$ is a bounded sequence and hence $\left\|\Phi_{\lambda, h}^{\prime}\left(u_{m}\right)\right\|_{*}\left\|u_{n}-u_{m}\right\| \rightarrow 0$ as $m, n \rightarrow \infty$. Finally by (6.1), $\lim _{n \rightarrow \infty} \Phi_{\lambda, h}\left(u_{n}\right)=c$, and so from (8.1) we can now conclude that $\left\{u_{n}\right\}$ is a Cauchy sequence in $X$. Thus $u_{n} \rightarrow u$ strongly in $X$. Returning to (6.1) and using Proposition 3.1 we obtain $\Phi_{\lambda, h}(u)=c$ and $\Phi_{\lambda, h}^{\prime}(u)=0$.

\section{Appendix}

If $\Gamma \in C^{2}([0, \infty))$, the partial differential equation in $(1.2)$ can be written as

$$
-\sum_{i, j=1}^{N} a_{i j}(u, \nabla u) \partial_{i j}^{2} u+\left\{\gamma\left(\frac{1}{2}\left[u^{2}+|\nabla u|^{2}\right]\right)-\gamma^{\prime}\left(\frac{1}{2}\left[u^{2}+|\nabla u|^{2}\right]\right)|\nabla u|^{2}-\lambda\right\} u=h
$$

where $a_{i j}(u, \nabla u)=\gamma\left(\frac{1}{2}\left[u^{2}+|\nabla u|^{2}\right]\right) \delta_{i j}+\gamma^{\prime}\left(\frac{1}{2}\left[u^{2}+|\nabla u|^{2}\right]\right) \partial_{i} u \partial_{j} u$.

The conditions (g1) and (g2) imply uniform ellipticity in the following sense.

For $z \in \mathbb{R}$ and $p, \xi \in \mathbb{R}^{N}$, setting $s=\frac{1}{2}\left[z^{2}+|p|^{2}\right]$, we have that

$$
\begin{aligned}
\sum_{i, j=1}^{\infty} a_{i j}(z, p) \xi_{i} \xi_{j} & =\gamma(s)|\xi|^{2}+\gamma^{\prime}(s)(p \cdot \xi)^{2} \\
& \geq \gamma(s)|\xi|^{2}+\gamma^{\prime}(s)|p|^{2}|\xi|^{2} \text { since } \gamma^{\prime}(s) \leq 0 \text { by }(\mathrm{g} 1) \\
& \geq \gamma(s)|\xi|^{2}+\gamma^{\prime}(s) 2 s|\xi|^{2}=\frac{1}{2} g^{\prime \prime}(t)|\xi|^{2} \text { where } t=\sqrt{s} \\
& \geq \rho|\xi|^{2} \text { by }(\mathrm{g} 2) .
\end{aligned}
$$

\section{References}

[1] Ambrosetti, A. and Rabinowitz, P. H.: Dual variational methods in critical point theory and applications, J. Funct. Anal., 14 (1973), 349-381 
[2] Arcoya, D. and Boccardo, L.: Critical points for multiple integrals of Calculus of Variations, Arch. Rat. Mech. Anal., 134 (1996), 249-274

[3] Cerami, G.: Un critero di esistenza per i punti critici su varietà illimitate, Ren. Acad. Sci. Let. Ist. Lombardo, 112 (1978), 332-336

[4] Cerami, G.: Sull' esistenza di autovalori per un problema al contorno non lineare, Ann. di Mat., 24 (1980), 161-179

[5] Bartolo, P., Benci, V. and Fortunato, D.: Abstract critical point theorems and application to some nonlinear problems with strong resonance at infinity, Nonlin. Anal. TMA, 7 (1983), 981-1012

[6] Ghoussoub, N.: A min-max principle with a relaxed boundary condition, Proc. AMS, 117 (1993), 439-447,

[7] Gilbarg, D. and Trudinger, N.S.: Elliptic Partial Differential Equations of Second Order, 2nd Edition, Springer, Berlin 1998

[8] Rabier, P.J.: On the Ekeland-Ghoussoub-Preiss and Stuart criteria for locating Cerami sequences, preprint.

[9] Struwe, M.: Variational Methods, Springer, Berlin, 1996

[10] Stuart, C.A.: Locating Cerami sequences in a mountain pass geometry, Comm. Appl. Anal. to appear

[11] Stuart, C.A. and Zhou, H-S.: Existence of guided cylindrical TM-modes in a homogeneous self-focusing dielectric, Ann. I. H. Poincaré Anal. Nonlin., 18 (2001) 69 - 96

[12] Stuart, C.A. and Zhou, H.-S.: Existence of guided cylindrical TM-modes in an inhomogeneous self-focusing dielectric, Math. Models Meth. Appl. Sci., 20 (2010), 1681-1719

[13] Zhang, K.: Mountain pass solutions for a double-well energy, J. Diff. Equats, 182 (2002), 490-510

\section{Acknowledgment}

It is with great pleasure that I record my sincere thanks to OxPDE, Oxford University for hospitality and support in the autumn of 2010. Most of the work for this paper was done during that visit.

C.A. Stuart

Section de mathématiques

Station 8

EPFL

CH 1015 Lausanne

Switzerland

e-mail: charles.stuart@epfl.ch

Received: March 3, 2011. 